\title{
Integrated analysis of the whole-transcriptome of sheep skeletal muscle reveals the ceRNA regulation network related to muscle fiber formation in sheep
}

\author{
Meiying Fang ( $\nabla$ meiying@cau.edu.cn ) \\ National Engineering Laboratory for Animal, China Agricultural University \\ Ran Cui \\ National Engineering Laboratory for Animal, China Agricultural University \\ Xiaolong Kang \\ Ningxia University \\ Yufang Liu \\ Hebei University of Engineering \\ Zhen Li \\ National Engineering Laboratory for Animal, China Agricultural University \\ Ximing Liu \\ National Engineering Laboratory for Animal, China Agricultural University \\ Shuheng Chan \\ National Engineering Laboratory for Animal, China Agricultural University \\ Yubei Wang \\ National Engineering Laboratory for Animal, China Agricultural University
}

\section{Research Article}

Keywords: Tan sheep, muscle fiber formation, transcriptome, ceRNA regulation network

Posted Date: February 10th, 2022

DOI: https://doi.org/10.21203/rs.3.rs-1256802/v1

License: (c) (i) This work is licensed under a Creative Commons Attribution 4.0 International License. Read Full License 


\section{Abstract}

Background: The different muscle fiber types are closely related to meat quality. Tan sheep is famous for the fur breed of China, and the meat quality is among the best in various sheep breeds. The mutton of Tan sheep have many characteristics including tender, juicy, nutritious and unique flavor. In this study, the high-throughput RNA sequencing was used to identify the expression profiles of coding and non-coding RNAs from longissimus dorsi and biceps femoris samples obtained from Tan sheep, Dorper sheep and their hybrid Dutanhan sheep.

Results: The results of immunofluorescence staining showed that the proportion of slow-twitch fibers in longissimus dorsi tissue in Tan sheep (about 11.247\%) was significantly higher than that in Dorper sheep (about 5.408\%) $(P<0.05$ ). A total of 819 difference expression (DE) IncRNAs, 354 DE circRNAs, $139 \mathrm{DE}$ mRNAs, and $36 \mathrm{DE}$ miRNAs were identified in high and low slow-twitch fiber ratio groups. Several key genes involved in signaling pathways that form different types of muscle fibers, including protein kinase AMP-activated catalytic subunit alpha 2 (PRKAA2), EF-hand domain family member B (EFHB), folliculin interacting protein 2(FNIP2) and L-lactate dehydrogenase A chain $(L D H A)$, had significantly different levels of expression in the two muscle tissues. Functional analysis suggested that the differentially expressed transcripts are involved in biological processes such as FoxO signaling pathways, AMPK signaling pathway and $\mathrm{Ca}^{2+}$ signaling pathway. There were 26 IncRNAs, 115 circRNAs, 21 miRNAs and 4 genes formed 70 mRNA-miRNA, 31 IncRNA-miRNA, and 158 circRNA-miRNA interaction relationships, suggesting that complex regulatory networks of coding and non-coding genes underlie the muscle fiber formation in Tan sheep. The functional prediction results showed that many of these DE circRNAs, such as novel_circ_0017336, novel_circ_0007039, novel_circ_0017132, competing to bind with miR-23a, might act as competing endogenous RNAs (ceRNAs) to control PRKAA2, EFHB and FNIP2 level, respectively. RT-qPCR validation for randomly selected 4 DEGS (PRKAA2, EGR1, MYL3 and FBXL5), 5 DEMs (miR-26a, miR-308-3p, miR-409-3p, miR-495-3p and miR-23a), and 4 DECs (circ_0017336, circ_0017132, circ_0017430, and circ_0007039) expression patterns were highly consistent with those obtained by RNA-Seq.

Conclusion: Our study provides a foundation for understanding how IncRNA, circRNA, miRNA and genes interact to regulate muscle fiber formation in Tan sheep, Dorper sheep and their hybrid. We also constructed IncRNA/circRNA-miRNA-gene interaction networks to elucidate the complex molecular mechanisms underlying muscle fiber formation. The results extend our knowledge about the diversity of meat trait among different domestic animals and provide a foundation for studying novel mechanisms that control meat trait in Chinese indigenous sheep.

\section{Introduction}

As a unique local sheep in Ningxia, China, Tan sheep possesses the characteristics of typical sheep in the northwestern arid and desertified grassland areas of China. The mutton of Tan sheep have many characteristics including tender, juicy, nutritious and unique flavor. As a cross between Black headed Persian and Dorset from South Africa, Dorper sheep is famous for its rapid muscle growth for producing a desirable carcass[1]. Dutanhan sheep is a cross population of Dorper sheep, Tan sheep and small-tailed Han sheep, whose meat quality indicators are between that of Dorper sheep and Tan sheep[2].

The proportion of slow-twitch fiber in sheep is positively correlated with various aspects of meat quality, such as tenderness, flavor and juiciness, and as such is one of the key traits related to consumer preference[3-5]. Skeletal muscle is composed of two types of muscle fibers that are classified as slow-twitch and fast-twitch fiber, which show different metabolic and contractile properties. Slow-twitch fibers exhibit a high oxidative capacity and a high mitochondrial content and are resistant to fatigue. In contrast, fast-twitch fibers display low oxidative metabolism and low mitochondrial content. With the deepening of research, more and more regulatory factors have been identified to be involved in the formation of slow-twitch fiber, including calcium/calmodulin-dependent protein kinase ( $\left.\mathrm{Ca}^{2+} / \mathrm{CaMKs}\right)$ signaling pathways[6-8], Ras-MAPK signaling pathway[9], Wnt signaling pathway[10], et al. However, more effective selection markers for slow-twitch fiber formation participated in the sheep needs to be found. Transcriptional profiling is a powerful approach for identifying transcripts and their expression patterns, especially more and more studies have shown that circRNAs are involved in transcriptional regulation [8] and cellular communication and signal transduction [10], and play a variety of important roles in muscle growth and development [9].

In this study, the aim of our work is to investigate the transcriptional patterns for coding genes and non-coding genes that may regulate different type of muscle fiber formation in Tan sheep, Dutanhan sheep and Dorper sheep. The results would provide insight into the complex molecular mechanisms underlying transcriptional network, which contributes to increase the ratio of slow-twitch fiber in actual production to improve the quality of sheep meat.

\section{Materials And Methods}

\section{Animal Sample Collection}


9 unrelated sheep (no common grandparents) were selected in 3 different groups (Tan sheep, Dorper sheep and Dutanhan sheep), which were raised in identical conditions in Ningxia, China. Sheep at the 8th month of growth were slaughtered in the same day. After slaughter, the longissimus dorsi and biceps femoris muscle samples were collected from each sheep and stored until use at $-80^{\circ} \mathrm{C}$ or in liquid nitrogen. Animal treatment and sample collection were approved by the Animal Welfare Committee of the State Key Laboratory of Agricultural Biotechnology, China Agricultural University, and in accordance with the Regulations on Administration of Affairs Concerning Experimental Animals, revised June 2004 (Ministry of Science and Technology).

\section{Immunofluorescence Staining}

Longissimus dorsi muscle was made into frozen sections. Tissue sections were fixed for $10 \mathrm{~min}$ in $4 \%$ paraformaldehyde at room temperature then $0.1 \%$ TritonX-100 for $10 \mathrm{~min}$. After blocked for $1 \mathrm{~h}$ using $1 \%$ BSA, tissue sections were labeled with primary antibodies $(\mathrm{MYH} 7,1 / 250)$ for overnight at $4^{\circ} \mathrm{C}$. Next day, tissue sections were washed 3 times with 1 xPBS and then stained with respective Alexa flour 488 secondary antibody $(1 / 250)$ for $1 \mathrm{~h}$ at room temperature. Tissue sections were then washed and $10 \mathrm{mg} / \mathrm{ml}$ Hoechst was added for $15 \mathrm{~min}$.

\section{RNA ExtractionखLibrary Preparation and Data Analysis}

Total RNA was isolated using TRIZOL $\circledast$ Reagent (Invitrogen, San Diego, CA, USA) according to the manufacturer's instructions. RNA quality was assessed using $1 \%$ agarose gels. RNA purity was determined using a K5500 Spectrophotometer (Kaiao, Beijing, China). RNA integrity and concentration was assessed using the RNA Nano 6000 Assay Kit and the Bioanalyzer 2100 system (Agilent Technologies, Foster, CA, USA).

After removed the ribosomal RNA with the Ribo-Zero rRNA kit (Epicentre, WI, USA), the RNA libraries (mRNAs, IncRNAs, and circRNAs) were generated with the mRNA-Seq Sample Preparation Kit (IIlumina, CA,USA). The library quality was measured with the Agilent Bioanalyzer 2100 system (Agilent Technologies) and then sequenced using the HiSeq 4000 platform (Illumina, San Diego, CA, USA) according to the vendor's recommended protocol. Raw data (raw reads) of fastq format were obtained by removing reads containing adapter, reads on containing ploy$\mathrm{N}$ and low quality reads from raw data. Reference genome (Oar_rambouillet_v1.0) and gene model annotation files were downloaded from genome website directly. Index of the reference genome was built using bowtie2 [11] and paired-end clean reads were aligned to the reference genome using HISAT2[12]. The mapped reads of each muscle sample were assembled by StringTie [12] in a reference-based approach. After this, we evaluated the assembled transcripts using five criteria to identify IncRNAs: (1) transcripts with exon number < 2 were removed; (2) transcripts with length $\leq 200$ bp were removed; (3) known no-IncRNA annotations were removed; (4) transcripts with Fragments Per Kilobase of exon per Million fragments mapped (FPKM) < 0.5 were removed; (5) coding-non-coding-index (CNCI)[13], coding potential calculator (CPC) [14], PFAM-scan[15], phylogenetic codon substitution frequency (PhyloCSF) [16] were used to distinguish mRNAs from IncRNAs. Those transcripts that were predicted to have coding potential by all of the above tools named as candidate set of novel protein-coding transcripts, whereas those without coding potential were named as novel IncRNAs. Cuffdiff was used to calculate FPKMs of both IncRNAs and coding genes in each sample[17]. Use find_circ[18] and CIRI2[19] two kinds of software to filter circRNA and the results were intersected according to the position of circRNA on the chromosome. IRES finder software was used to predict whether circRNA sequences have potential IRES elements.

Refer to the standard procedure, miRNA libraries were constructed and then the quality was assessed as above cDNA libraries. The libraries were then sequenced with Illumina HiSeq ${ }^{\text {Tw}} 2500$ system according to the vendor's recommended protocols. Clean reads were obtained after the removal of raw reads containing 5'adaptor, 3 'adaptor, no insertion sequence, and poly $(A)$ in small RNA fragments, as well as those shorter than 18nt, of known classes of RNAs (ribosomal RNAs, messenger RNAs, small nuclear RNAs, transfer RNAs, small nucleolar RNA, small cytoplasmic RNAs, and repeats), and of low quality. Bedtools (https://bedtools.readthedocs.io/) was used to search for known miRNAs by matching them to entries in miRBase20.0 (http://www.mirbase.org/). After excluding reads that were mapped to known miRNAs, miRDeep2[20] was used to analyze the remaining reads to predict novel miRNAs. The expression amount of known and new miRNA and circRNA in each sample was counted, and the expression quantity was normalized by TPM[21].

\section{GO and KEGG enrichment analysis}

DESeq [22]was used for differential gene expression analysis of transcripts among three groups. only the transcripts with significant differences $(P$-values $<0.05)$ and $\mid \log _{2} \mathrm{FCl}>1$ were classified as DE transcripts. Gene ontology $(G 0)$ enrichment analysis of DE mRNAs, DE circRNAs parental genes , DE IncRNAs and DE miRNAs target genes were conducted using the GO-seq R package[23], correcting for gene length bias. Kyoto Encyclopedia of Genes and Genomes (KEGG) is a database resource for understanding high-level functions and utilities of the biological system[24], such as the cell, the organism and the ecosystem, from molecular-level information, especially large-scale molecular datasets generated by genome sequencing and other high-throughput experimental technologies. KOBAS v3.0[25] was used to test the 
statistical significance for the enrichment of DEGs or DE circRNAs parental genes or targets of DE IncRNA and DE miRNAs in KEGG pathways. Only the $p<0.05$ was considered statistically significant.

\section{LncRNA/circRNA-miRNA-mRNA Network Analysis}

For the cis-acting prediction, coding genes $10 \mathrm{~kb}$ upstream and downstream of IncRNA were searched as cis-acting regulatory targets. For the trans-acting prediction, the expression of the IncRNA was determined to be not related with the location of the mRNA but co-expressed with it. The expressed correlation was calculated between IncRNAs and coding genes using Pearson's correlation coefficients $(r>0.90$ or $r<-0.90)$ as a classifier. The prediction of miRNA target genes was performed using miRanda[26], PITA[27] and RNAhybrid[28]. CeRNA hypothesis RNA transcripts can crosstalk by competing for common miRNAs, with miRNA response elements as the foundation of this interaction[29]. IncRNA/circRNA-miRNA-mRNA pairs were constructed with IncRNA/circRNA as decoy, miRNA as the core, and mRNA as the target. The IncRNA, circRNA, miRNA and mRNA interactions were constructed and visualized using Cytoscape[30].

\section{Quantitative Polymerase Chain Reaction}

To evaluate the reliability of the transcript expression data obtained by RNA-Seq. Quantitative Polymerase Chain Reaction (qPCR) were carried out with the longissimus dorsi tissue. Using FastKing gDNA Dispelling RT SuperMix and SuperReal PreMix Plus (Tiangen, China), experiments were conducted for mRNA detection. Using miRcute Plus miRNA first-strand cDNA Kit and miRcute Plus miRNA qPCR Kit (Tiangen, China), experiments were conducted for miRNA detection. Using RT primers and Custom gene qRT-PCR Quantitation Kit (GenePharma, China), experiments were conducted for circRNA detection. To verify the back-splicing junction of circRNA, the RNase R- and control reaction (without RNase R) were prepared and reverse transcribed into CDNA to amplify each circRNA with primers following previously method[31, 32], then the products were sequenced to find the back-splicing sites. The GAPDH and U6 small nuclear RNA genes were selected as the endogenous control genes (all primers are shown in Table S12). All qPCR validations were carried out with three biological replicates and triplicate reactions for each sample. After amplification, the products were confirmed by agarose gel electrophoresis and Sanger sequencing, the relative transcript abundance was calculated using $2^{-\Delta \Delta C t}$ method.

\section{Statistical Analysis}

Data were expressed as means \pm standard deviation (SD). Significance was analyzed using one-way analysis of variance (ANOVA) to test homogeneity of variances via Levene's test, followed by Student's t-test. Calculations were conducted using SAS version 9.0 (SAS, Cary, NC, USA). Differences were considered to be statistically significant for $P$-values $<0.05$.

\section{Results}

\section{The ratio of fast and slow muscles fiber in longissimus dorsi tissue in different populations}

The results of immunofluorescence staining showed that the proportion of slow-twitch fibers in longissimus dorsi tissue in Tan sheep was about $11.247 \%$, and that in Dorper sheep was about 5.408\%. Compared with Dorper sheep, the proportion of slow-twitch fibers was significantly higher in Tan sheep $(P<0.05)$, and the increase ratio accounted for about $51.9 \%$ of Tan sheep(Fig. 1$)$.

\section{Overview of RNA sequencing}

To assess the genes involved in muscle fiber formation, longissimus dorsi (LD) and biceps femoris (BF) tissues were collected from the 8month-old Dorper sheep, Tan sheep and Dutanhan sheep for the whole-transcriptome profiling of all mRNAs and noncoding RNAs. For the RNA sequencing libraries, an average of 95.74 million clean reads were obtained from the 18 samples tested, and $83.55-90.07 \%$ of these reads were uniquely aligned to the reference genome Oar_rambouillet_v1.0. All 18 samples had at least $85 \%$ reads equal to or exceeding Q30 (Table S1-1). In addition, for the small RNA-Seq libraries, an average of 16.02 million clean reads were obtained (Table S1-2). Moreover, 107 mRNAs were differently expressed, which included $47 \mathrm{BF}$ tissue-specific and $37 \mathrm{LD}$-specific mRNAs, respectively. Apart from mRNAs, 224 known IncRNAs and 22373 novel IncRNAs were identified from the RNA-seq data by blasting to IncRNAs in the NCBI database and performing CNCl, CPC2 and PfamScan analysis (Fig.S1). Additionally, 737 IncRNAs were differently expressed, of which 216 BF tissue-specific and 242 LDspecific. Of these DE IncRNAs, 37.9\% were expressed in both types of tissues. Furthermore, 238 miRNAs were identified and 32 miRNAs were 
differently expressed, of which 22 were BF tissue-specific and 4 were LD-specific. Finally, 3648 circRNAs were identified and 348 circRNAs were differently expressed, of which 188 and 135 were found to be BF tissue-specific and LD-specific, respectively (Table S2-1, S2-2).

\section{DE mRNAs related to muscle fibers formation}

First, DEGs between the LD and BF tissues from 8-month-old Dorper sheep, Tan sheep and Dutanhan sheep were screened, where upon 149 DEGs were found, and their $\log _{2}$ FC values were presented as Volcano Plot pictures, which ranged from -20 to 20 . Among all of these mRNAs, 25 (13 up-regulated, 12 down-regulated) and 35 (24 up-regulated, 11 down-regulated) DE mRNAs were identified in the D_L/T_L and D_B/T_B, 32 and 42 were identified in DTH_L/T_L and DTH_L/D_L, and 21 and 41 were identified in DTH_B/T_B and DTH_B/D_B, respectively(Fig.2a). The amount of DE mRNAs in BF and LD tissues was basically the same, implying that they might participate in the equally important response process under meat quality.

To explore the functions of these DE mRNAs, GO annotation and KEGG enrichment analysis were performed (Fig.2b and c). GO annotation analysis showed that these genes were involved in calcium ion binding (G0:0005509), cellular response to calcium ion (GO:0071277), ATPase inhibitor activity (GO:0042030), oxidation-reduction process (G0:0055114). KEGG enrichment analysis showed that muscle fiber formation, metabolic pathways, cAMP signaling pathway were significantly enriched, and these terms of DE mRNAs possible have primarily participated in meat quality. Among them, DEGs included protein kinase AMP-activated catalytic subunit alpha 2 (PRKAA2, log 2 Fold change $\left(\log _{2} F C=8.11\right)$, EF-hand domain family member $B\left(E F H B, \log _{2} F C=2.43\right)$, L-lactate dehydrogenase $A$ chain $\left(L D H A\right.$, $\left.\log _{2} F C=3.35\right)$, folliculin interacting protein 2 (FNIP2, $\left.\log _{2} \mathrm{FC}=3.05\right)$, which are related to pathways associated with muscle fiber formation: the cellular response to calcium ion, calcium ion binding, oxidation-reduction process and ATPase inhibitor activity, respectively (Table S4).

In addition, 3 DEGs(ZIC1 $\square Y$ Y $3 \square A S A H 1)$ were co-differentially expressed in both LD and BF tissues(Fig.S3a), all of which had known functions in muscle fibers growth and development. These differentially expressed transcripts may be related to the different function during the development of these two tissues, which are worthy of future analysis.

\section{DE IncRNAs related to muscle fibers formation}

Comparison of the genomic characterizations of the IncRNAs with mRNAs showed that their transcripts were similar in length distribution; for exon number, a higher number of IncRNAs had 2 to 4 exons; in addition, IncRNAs had a shorter ORF length and lower FPKM value (Fig.3a and b). The $\log _{2}$ FC values of DE IncRNAs both ranged from -20 to 20 in the LD and the BF tissue. Among all of these IncRNAs, 214 (113 upregulated, 101 down-regulated) and 172 (144 up-regulated, 28 down-regulated) DE mRNAs were identified in the D_L/T_L and D_B/T_B, 210 and 251 were identified in DTH_L/T_L and DTH_L/D_L, and 161 and 206 were identified in DTH_B/T_B and DTH_B/D_B , respectively(Fig.3c).

To reveal the potential functions of the 838 identified IncRNAs in muscle fiber formation, two independent algorithms-cis (genomic location) and trans (expression correlation) -were performed to predict the target genes of the IncRNAs. In total, 720 were targets of 2154 cisacting IncRNAs, and 163 showed the most correlated co-expressed with 446 trans-acting IncRNAs. GO annotation of cis-IncRNAs were significantly enriched in skeletal muscle fiber development (G0:0048741), skeletal muscle contraction (G0:0003009), Negative regulation of glycolysis (GO:0006110), ATPase activity (G0:0042623), etc.; KEGG enrichment were significantly enriched in the metabolic pathway, Muscle atrophy, etc (Table S6). GO annotation of trans-IncRNAs were significantly enriched in positive regulation of skeletal muscle tissue development (GO:0048643), muscle cell differentiation (GO:0042692), etc.; KEGG enrichment were significantly enriched in Glycolysis, TCA cycle, Wnt signaling pathway, etc ((Fig.3 d and e). As a preliminary exploration of the functional implications of the DELs across genomes, we investigated whether IncRNAs were co-regulated with the DEGs during muscle fiber formation. Interestingly, in both LD and BF tissues, we observed that the cis-IncRNA LNC_002028 targeted EFHB as its DE target gene, whereas the three trans-IncRNAs LNC_003248, LNC_003249, LNC_013114 targeted PRKAA2 as their DE cotarget genes. In addition, 4 DELs(LNC_003158ロLNC_0152910LNC_015292 differentially expressed in both LD and BF tissues(Fig.S3b).

\section{DE miRNAs related to muscle fibers formation}

In the present study, raw reads were generated 42 DE miRNAs from D_L, DTH_L, T_L, D_B, DTH_B and T_B small RNA libraries, respectively. The lengths of most clean reads were $20-24$ nt (Fig.4a). Small RNA classification showed that $68.65 \%$ of clean reads were positive and negative chains of exon/intron, and there were also $8.93 \%$ miRNA, and $22.42 \%$ of other types (Fig. $4 \mathrm{~b}$ ). Among all of these miRNAs, 4 (3 upregulated, 1 down-regulated) and 12 (7 up-regulated, 5 down-regulated) DE miRNAs were identified in the D_L/T_L and D_B/T_B, 2 and 7 were identified in DTH_L/T_L and DTH_L/D_L, and 2 and 24 were identified in DTH_B/T_B and DTH_B/D_B, respectively (Fig. 4c, Table S8). In addition, oar-miR-10b were co-differentially expressed in both LD and BF tissues (Fig.S3c). GO annotation and KEGG enrichment analysis of 
the targets showed that most of them were enriched to metabolic pathways (Fig. $4 \mathrm{~d}$ and e). In addition, 6 miRNAs (oar-miR-21, oar-miR-26a, oar-miR-27a, oar-miR-29b, oar-miR-409-3p, novel_67) were expressed in both LD and BF tissues, and the expression trend was the same (Table S9).

\section{DE circRNAs related to muscle fibers formation}

A total of 3651 circRNAs were identified in this study, and circRNAs were defined with at least two independent reads crossing the backsplice junction.Among the 3651 circRNAs we studied, circRNAs were more likely to be produced from exons, 3141 circRNAs were generated from exons of 1810 genes, 197 circRNAs were generated from intronic regions of 175 genes, and the rest were intergenic circRNA according to our statistics (Fig. 5a).CircRNAs were mostly located on chromosome 1 and ranged in length from 192nt to 93278nt (Fig. 5b). Read counts across backsplice junction sites were normalized to transcripts per million (TPM) (Fig. 5c). The source sites of circRNAs (including exons, introns, and intergenic regions) were different, but no significant changes were found in the lengths of circRNAs from different sources (Fig. $5 d$ ).In addition, the expression correlation between circRNAs and their host genes was also detected based on the linear splicing sites of the parental gene and the reverse splicing sites of the circRNAs. The Pearson correlation coefficient between circRNAs and their host genes was 0.046 , indicating a very low correlation (Fig. 5e).Then, the coefficient of variance of each circRNA and its parental gene was calculated as a measure of the variance of the expression level, and it was found that circRNAs may be easily regulated by multiple factors and therefore have higher expression variability (Fig. 5f).Interestingly, it was found that circRNAs had extremely high potential to encode proteins, and 4611 internal ribosome entry sites (IRES) were predicted, and the highest score could be as high as 0.997 (Table S10).

Among all of these circRNAs, 91(46 up-regulated, 45 down-regulated) and 95 (36 up-regulated, 59 down-regulated) DE circRNAs were identified in the D_L/T_L and D_B/T_B, 92 and 79 were identified in DTH_L/T_L and DTH_L/D_L, and 70 and 39 were identified in DTH_B/T_B and DTH_B/D_B, respectively (Fig.5h). GO annotation analysis of the targets showed that most of them were related to myofibril (G0:0030016), glycogen metabolic process (G0:0005977), etc.; KEGG enrichment were enriched in FoxO signaling pathways (including CDKN1A, $P R K A A 1$, FBXO32, PRKAG3 gene), AMPK signaling pathway (including PRKAG3, PGC-1a, SCD, MLYCD, PRKAA1, LIPE, PRKAA2 genes), Ca ${ }^{2+}$ signaling pathway (including ORA/1, TACR1, ATP2B2, CACNA1D, HTR2A genes), etc (Fig.5 g and i, TableS11). In addition, novel_circ_0009997 were codifferentially expressed in both LD and BF tissues (Fig.S3d).

\section{Construction of the ceRNA regulatory network}

It has been shown that mRNAs, IncRNAs, and circRNAs may act as ceRNAs, which regulate gene function via miRNA in various processes, suggesting that ceRNAs and their miRNAs may be co-regulated in muscle fiber formation. On the basis of the data of the DE mRNA, IncRNA, circRNA, and miRNA transcripts, a ceRNA network was constructed consisting of 4 DEGs, 21 DEMs, 115 DECS, and 26 DELs, and obtained 70 mRNA-miRNA, 31 IncRNA-miRNA, and 158 circRNA-miRNA interaction relationships (Fig.6, Fig.S2). Within the network, it was found that PRKAA2, EFHB, FNIP2 and $\angle D H A$ may be the crucial genes mediated by noncoding RNAs for regulating muscle fiber formation. The functional prediction results showed that many of these DE circRNAs, such as novel_circ_0017336, novel_circ_0007039, novel_circ_0017132, competing to bind with miR-23a, might act as ceRNAs to control PRKAA2, EFHB and FNIP2 level, respectively.

\section{Validation of gene expression by RT-qPCR}

Validation of the RNA-seq results was carried out using the qPCR for 4 DEGS (PRKAA2, EGR1, MYL3 and FBXL5), 5 DEMs (miR-26a, miR-3083p, miR-409-3p, miR-495-3p and miR-23a), and 4 DECs (circ_0017336, circ_0017132, circ_0017430, and circ_0007039) (Fig.7 a, b and d). The expression of these selected transcripts was significantly different in the LD tissue in Tan sheep and Dorper sheep, with the expression patterns being highly consistent with those obtained by RNA-Seq. It's worth mentioning that the backsplice junctions of circRNAs were confirmed before the validation, due to the circular structure, the circRNAs were more resistant to digestion by RNase R treatment, and the back-splicing sites were verified by Sanger sequencing (Fig.7c). The results indicated the high reproducibility and reliability of the gene expression profiles obtained in our study.

\section{Discussion}

As is widely known, the muscle fiber formation is one of the polygenic traits in sheep that is an important determinant of meat quality characteristics. In general, the diameter of slow-twitch fiber is thinner than that of fast-twitch fiber, while the density of slow-twitch fiber is larger than that of fast-twitch fiber, and its proportion is positively correlated with meat quality. There are some differences in histological characteristics of muscle fibers among different populations of sheep. In previous studies, compared LD tissues with Dutanhan sheep and Dorper sheep, Tan sheep had the thinnest muscle fiber diameter, while the muscle fiber density of Tan sheep was significantly greater than that 
of Dorper sheep and Dutanhan sheep [2]. In this study, we calculated the ratio of fast and slow muscle fibers in LD tissue by immunofluorescence staining, whereupon it was found that Tan sheep has a higher proportion of slow muscle fibers than Dorper sheep. This prompted us to choose LD and BF two muscle tissues in different groups for the whole transcriptome profile analysis to identified DEGs and DE non-coding RNAs. These changes may be closely related to the formation of slow muscle fibers in sheep.

The sheep used in this study are 8-month-old population that have attained consistency in appearance, reproductive and production performances. 139 DEGs were identified in the muscle tissues of three groups, many of which have known functions in muscle fiber formation. For example, PRKAA2 encodes the AMPKa2 subunit, and the AMPKa2 subunit is a catalytic subunit of AMPK, which participates in the activation of AMPK protein. AMPK family gene polymorphism plays a role in skeletal muscle gene expression differences and effects on meat quality [7, 33-35]. The expression level of PRKAA2 in Tan sheep is higher than that in Dorper sheep, which may also promote the formation of slow muscle fiber by increasing the level of energy metabolism. Moreover, the expression of FNIP2 was in consistent with the increased trend of slow muscle fiber, whereas $E F H B, \angle D H A$ showed the opposite trend, indicating that the former gene acts as positive regulators in slow muscle fiber formation whereas the latter genes act as negative regulator in this process. FNIP2 is involved in the regulation of ATPase inhibitor activity. It has been reported that FNIP may regulate AMPK pathway via direct or indirect pathways, thereby regulating mitochondrial biosynthesis and muscle fiber types. EFHB participates in the regulation of calcium ion binding, the transcription factors of mitochondrial nuclear-encoded genes are activated in skeletal muscle by $\mathrm{Ca}^{2+}$-dependent transport pathways, and then cause the formation of muscle fibers. $L D H A$ is involved in the regulation of oxidation-reduction process and lactate dehydrogenase activity. Besides, 3 DEGs (ZIC1, MYL3, ASAH1), 4 DELs (LNC_003158, LNC_015291, LNC_015292, LNC_015293), 1 DEMs(oar-miR-10b) and 1 DECs(novel_circ_0009997) were co-differentially expressed in both LD and BF tissues, many of which had known functions in muscle fibers growth and development. For instance, studies have shown that ZIC1 is differently expressed in longissimus dorsi and semitendinosus of the Wuzhishan pig, and it can play a role in Myf5 regulation and somite myogenesis[36]. MYL3, myosin light chain 3, has been identified as candidate function gene in muscle development in Dorper and small-tailed Han sheep[37]. While mutations in ASAH1 will cause the occurrence of muscular atrophy in rare diseases[38]. These differential expression transcripts may be related to the different function of these genes during the development of these two tissues, which are also worthy of future analysis.

Although biological processes, cellular composition, and molecular functions finally depend on the expression of protein-coding genes, over the last twenty years, the concept has been transformed that the expression of protein-coding genes in turn depend on an intricate transcriptional network, including a variety of non-coding RNAs[39-41]. A very abundant classe of ncRNAs ( 270,000 transcripts) are IncRNAs, which are characterized by transcripts of 200nt or longer. They share many features with mRNA, including polyadenylation, 5 capping and splicing, but most are not translated into protein. IncRNAs could play a major role in many physiological and pathological conditions through trans and cis activities, and prediction of IncRNA target genes by these two independent algorithms as well as bioinformatics analysis will help to identify which term IncRNAs are participated in and further showing potential function. Our results showed that IncRNAs obtained in this study may regulate pathways related to muscle fiber formation via trans and cis activities (such as skeletal muscle fiber development, Negative regulation of glycolysis, ATPase activity). In addition, our results also indicated that the cis-IncRNA, LNC_002028, and three transIncRNAs, LNC_003248, LNC_003249, and LNC_013114, also regulated two important candidate genes, EFHB and PRKAA2, respectively. On the basis of these results, we suspected that one of the main roles of these 4 IncRNAs is to regulate the muscle fiber formation in sheep, and further highlighting of their detailed mechanisms in this process would be fertile ground for future investigation.

Besides, miRNAs are small ( 22nt) trans-acting functional RNAs involved in silencing and post-transcriptional regulation of gene expression[39]. The results on the DEMs and DECs in muscle tissue indicated that they were significantly enriched in muscle fiber formation pathways, for instance, FOXO signaling pathway, $\mathrm{Ca}^{2+}$ signaling pathway, AMPK signaling pathway, contraction fiber, glycogen metabolic process. From the constructed ceRNA network, we found several miRNAs, including substantially down-regulated miR-409-3p and up-regulated miR-23a, miR-26a, miR-27a, miR-382-3p, novel-67 and miR-495-3p around PRKAA2. Studies have shown that miR-23a down-regulates the translation of mabbx/atrogin-1 and MuRF1 (mediated atrophic protein degradation) to promote muscle atrophy[40]; meanwhile, miR-23a inhibits myocyte differentiation by down-regulating $M Y H C$ gene expression[41]; A similar research of miR-23a was carried in Angus cattle at the 80 fetus ( 90 days old) and adult (24 months old) stages, circMYBPC1 was identified differently expressed and interacted with muscle genes MYHC through directly binding miR-23a[42]; miR-26a promotes myoblast differentiation by down-regulating the expression of its target gene Ezh2[43]. Our miRNA-mRNA interaction analyses identified a much large number of predicted miRNA-mRNA pairs in the comparison of Dorper sheep, Dutanhan sheep and Tan sheep, suggesting that Tan sheep have a greater ability to regulate the slow muscle fiber forming environment. CircRNA is a novel type of non-coding RNA, which exhibits high stability, abundance, tissue/stage specificity, and evolutionary conservation [9]. All of these 3651 DECs are new circRNAs that have not been discovered before in sheep, and there is little information or data obtained from published databases. In our study, the functional prediction results also showed 11 DE circRNAs, including novel_circ_0017336, novel_circ_0007039, novel_circ_0017132, competing to bind with miR-23a, might act as ceRNAs to control PRKAA2, EFHB and FNIP2 level, respectively, which side verifies the importance of miR-23a not only in its own function, but also acting as a bridge in the ceRNA mechanism of muscle fiber formation. GO and KEGG pathway analysis were constructed to the target genes involved in circRNA- or IncRNA-associated

Page $7 / 18$ 
ceRNA. Interestingly, GO annotation showed that associated genes are all enriched in the same term: regulation of skeletal muscle tissue growth. Further studies on $A N K R D, U C P 1, A D R B 3$ and other genes in this pathway are also necessary.

In this study, strict constraints were applied to select the most possible circRNA- or IncRNA-associated ceRNA networks that are involved in muscle fibers formation. For example, we selected ceRNA networks that participated in the control of crucial muscle gene related to signaling pathway. 4 DEGs, 115 DECs, and 26 DELs were connected with one another through the 21 DEMs, a total of 70 mRNA-miRNA, 31 IncRNAmiRNA, and 158 circRNA-miRNA interaction relationships were obtained. This indicated that muscle fiber formation in sheep is jointly regulated by these factors and results from a balance level of transcripts expression. We speculate that most of these factors may play a key role in the regulation of muscle fiber formation, these possibilities are worthy of further research efforts.

\section{Conclusion}

Our review gives a complete scene of the distinctions in the entire transcriptome profiles of LD and BF tissues among three gatherings in sheep. 4 DEGs were recognized connected with muscle fiber arrangement among various gatherings, including PRKAA2, EFHB, FNIP2, LDHA. Moreover, a few DE IncRNAs, miRNAs, and circRNAs were found in muscle tissue firmly connected with a few muscle fiber arrangement pathways. Based on the differently expressed records, a ceRNA regulatory network was developed which contained 4 DEGS, 115 DECS, 26 DELS, 21 DEMS and 259 connections. Our discoveries have distinguished potential regulators and molecular regulatory networks that might be associated with muscle fiber arrangement in sheep, and gave an establishment to future examinations on the molecular mechanisms underlying muscle fiber development.

\section{Abbreviations}

ASAH1: N-acylsphingosine amidohydrolase 1; ATP2B2: ATPase plasma membrane $\mathrm{Ca}^{2+}$ transporting 2; ATP6VOA1: ATPase $\mathrm{H}^{+}$transporting V0 subunit a1; CACNA1D: calcium voltage-gated channel subunit alpha1 D; CDKN1A: cyclin dependent kinase inhibitor 1A; EGR1: early growth response 1; FBXL5: F-box and leucine rich repeat protein 5; FBXO32: F-box protein 32; MYL3: myosin light chain 3; HDAC5: histone deacetylase 5; HTR2A: 5-hydroxytryptamine receptor 2A; LIPE: lipase E; MEF2: Myocyte enhancer factor 2; MLYCD: malonyl-CoA decarboxylase; ORAI1: ORAI calcium release-activated calcium modulator 1; PGC-1a: PPARG coactivator 1 alpha; PRKAA1: protein kinase AMP-activated catalytic subunit alpha 1; PRKAG3: protein kinase AMP-activated non-catalytic subunit gamma 3; SCD: stearoyl-CoA desaturase; SIRT1: sirtuin1; TACR1: tachykinin receptor 1; ZIC1: Zic family member 1;

\section{Declarations}

\section{Acknowledgements}

The authors gratefully thank Dr. Xiaolong Kang at Ningxia University (Yinchuan, China) for taking technical support.

\section{Authors' contributions}

MYF and RC conceived this study, and designed and supervised the experiments; RC, XML, SHC and XLK performed the experiments; RC, ZL and YBW conducted the data analysis and prepared figures and Tables. RC and YFL wrote the manuscript. All of the authors reviewed and approved the manuscript.

\section{Funding}

This work was financially supported by the Program of Agricultural Breeding in Ningxia Hui Autonomous Region (No. nxnyyz20150101).

\section{Availability of data and materials}

The data supporting the conclusions of this article can be found in Tables S3,S4,S5,S6,S7,S8,S9,S10. The RNA sequencing data generated during the current study has been uploaded in the NCBI BioSample database (https://www.ncbi.nlm.nih.gov/biosample/?term). The accession 
numbers of RNA-seq data of the 18 samples are 25271312 25271329, respectively. The accession number for RNA-seq data deposited in NCBI BioProject is PRJNA800627. The genome was obtained from NCBI using the accession number: Oar_rambouillet_v1.0.

\section{Ethics approval and consent to participate}

Animal care and experiments were conducted according to the Regulations for the Administration of Affairs Concerning Experimental Animals (Ministry of Science and Technology, China; revised June 2004) and were approved by the animal welfare committee of the State Key Laboratory for Agro-biotechnology of China Agricultural University (approval number XK257). Our study is reported in accordance with the ARRIVE (Animal Research: Reporting In Vivo Experiments) guidelines.

\section{Consent for publication}

Not applicable.

\section{Competing interests}

The authors declare that they have no competing interests.

\section{Author details}

1 Department of Animal Genetics and Breeding, National Engineering Laboratory for Animal Breeding, MOA Laboratory of Animal Genetics and Breeding, College of Animal Science and Technology, China Agricultural University, No. 2 Yuanmingyuan West Rd, 100194 Beijing, People's Republic of China.

2 College of Agriculture, Ningxia University, Yinchuan 750021, People's Republic of China.

3 College of Life Sciences and Food Engineering, Hebei University of Engineering, Handan 056021, People's Republic of China.

\section{References}

1. C. Milne: The history of the Dorper sheep. Small Ruminant Research2000, 36(2): 99-102.

2. Z Wen, L Xinhai, Z Tianwan,et al: Comparative analysis of meat performance and meat quality characteristics of four sheep groups in Ningxia. Northwest Agricultural Journa/2020, 29(08).

3. L. Su, H. Li, X. Xin,et al: Muscle Fiber Types, Characteristics and Meat Quality. 2013, 2203(634-638):1267.

4. W. Kaiqi, H. Weiran, L. Yi,et al: Fiber characteristics and meat quality of different muscular tissues from slow-and fast-growing broilers. Poultry Science2022, 101(1):101537.

5. H. Weiran, W. Kaiqi, G. Tiantian, et al: Effect of muscle fiber characteristics on meat quality in fast- and slow-growing ducks. Poultry science2021, 100(8):101264.

6. E.R. Chin: Role of Ca2+/calmodulin-dependent kinases in skeletal muscle plasticity. Journal of Applied Physiology2005, 99(2):414-423.

7. B. Viollet, F. Andreelli, S.B. Jrgensen, et al: Physiological role of AMP-activated protein kinase (AMPK): insights from knockout mouse models. Biochemical Society Transactions2003, 31(1):216.

8. E.R. Chin, E.N. Olson, J.A. Richardson,et al: A calcineurin-dependent transcriptional pathway controls skeletal muscle fiber type. Genes Dev1998, 12(16):2499-2509.

9. M. Murgia, A.L. Serrano, E. Calabria,et al: Ras is involved in nerve-activity-dependent regulation of muscle genes. Nature Cell Biology2000, 2(3):142-147.

10. K. Kuroda, S. Kuang, M.M. Taketo, et al: Canonical Wnt signaling induces BMP-4 to specify slow myofibrogenesis of fetal myoblasts. Skeletal Muscle2013, 3(1):5.

11. B. LangmeadandS.L. Salzberg:- Fast gapped-read alignment with Bowtie 2. 2012, 9(4):359.

12. M. Pertea, D. Kim, G.M. Pertea,et al: Transcript-level expression analysis of RNA-seq experiments with HISAT, StringTie and Ballgown. Nature Protocols2016, 11(9):1650-1667. 
13. L. Sun, H. Luo, D. Bu,et al: Utilizing sequence intrinsic composition to classify protein-coding and long non-coding transcripts. Nucleic Acids Research2013(17):e166-e166.

14. L. Kong, Y. Zhang, Z. Ye,et al: CPC: assess the protein-coding potential of transcripts using sequence features and support vector machine. Nucleic acids research2007, 35(Web Server issue):W345-W349.

15. R.D. Finn, J. Tate, J. Mistry, et al: The Pfam protein families database. Nucleic Acids Research2008, 32(1):D138.

16. M. Kellis: PhyloCSF: a comparative genomics method to distinguish protein coding and non-coding regions. Bioinformatics2011, 27(13):i275-i282.

17. C. Trapnell, B.A. Williams, G. Pertea,et al: Transcript assembly and quantification by RNA-Seq reveals unannotated transcripts and isoform switching during cell differentiation. Nature Biotechnology, 28(5):511-515.

18. Circular RNAs are a large class of animal RNAs with regulatory potency. Nature2013, 495(7441):333-338.

19. Y. Gao, J. ZhangandF. Zhao: Circular RNA identification based on multiple seed matching. Briefings in Bioinformatics2017(5):5.

20. M.R. Friedlnder, S.D. Mackowiak, N. Li,et al: miRDeep2 accurately identifies known and hundreds of novel microRNA genes in seven animal clades. Nucleic Acids Research2012(1):37-52.

21. L. Zhou, J. Chen, Z. Li,et al: Integrated Profiling of MicroRNAs and mRNAs: MicroRNAs Located on Xq27.3 Associate with Clear Cell Renal Cell Carcinoma. Plos One2010, 5(12):e15224.

22. W. Likun, F. Zhixing, W. Xi,et al: DEGseq: an R package for identifying differentially expressed genes from RNA-seq data. Bioinformatics (Oxford, England)2010, 26(1):136-138.

23. M. Young, M.J. Wakefield, G.K. Smyth,et al: Gene ontology analysis for RNA-seq: accounting for selection bias. Genome Biology2010, 11(2):R14-R14.

24. K. Minoru, A. Michihiro, G. Susumu, et al: KEGG for linking genomes to life and the environment. Nucleic Acids Research2008, 36(Database issue):480-484.

25. X. Chen, X. Mao, J. Huang,et al: KOBAS 2.0: a web server for annotation and identification of enriched pathways and diseases. Nucleic Acids Research2011, 39(Web Server issue):316-322.

26. A. Enright, B. John, U. Gaul,et al: MicroRNA targets in Drosophila. Genome Biology2003, 5(11):R1.

27. R. Marc, S. Peter, H. Matthias, et al: Fast and effective prediction of microRNA/target duplexes. RNA (New York, NY)2004, 10(10):15071517.

28. R. Ventsislav, B. Vesselin, M.I. Nikiforov, et al: Microlnspector: a web tool for detection of miRNA binding sites in an RNA sequence. Nucleic acids research, 33(Web Server issue):W696-W700.

29. L. Salmena, L. Poliseno, Y. Tay, et al: A ceRNA Hypothesis: The Rosetta Stone of a Hidden RNA Language? Cel/2011, 146(3):353-358.

30. S. Michaele, O. Keiichiro, R. Johannes, et al: Cytoscape 2.8: new features for data integration and network visualization. Bioinformatics (Oxford, England)2011, 27(3):431-432.

31. P.A. C.andG. Myriam: Detection and Analysis of Circular RNAs by RT-PCR. Bio-protoco/2018, 8(6):e2775.

32. L. Heng, H. Yin, Y. Jing, et al: Profiles analysis reveals circular RNAs involving zebrafish physiological development. Journal of cellular physiology2019, 234(9):15922-15933.

33. H.D. Grahame: Sensing of energy and nutrients by AMP-activated protein kinase. The American journal of clinical nutrition2011, 93(4):891S-896.

34. H. Takemori, Y.K. Hashimoto, J. Nakae, et al: Inactivation of HDAC5 by SIK1 in AICAR-treated C2C12 Myoblasts. Endocrine Journa/2009, 56(1):121-130.

35. C. Xiaoling, G. Yafei, J. Gang, et al: Ferulic acid regulates muscle fiber type formation through the Sirt1/AMPK signaling pathway. Food \& function2019, 10(1):259-265.

36. H. Pan, M.K. Gustafsson, J. Aruga,et al: A role for Zic1 and Zic2 in Myf5 regulation and somite myogenesis. Developmental Biology2011, 351(1):120-127.

37. Z. Chunlan, W. Guizhi, W. Jianmin,et al: Characterization and comparative analyses of muscle transcriptomes in Dorper and small-tailed Han sheep using RNA-Seq technique. PLoS One2013, 8(8):e72686.

38. R. Guido, V. Pierangelo, P. Antonella, et al: Spinal muscular atrophy associated with progressive myoclonic epilepsy: A rare condition caused by mutations in ASAH1. Epilepsia2015, 56(5):692-698.

39. D.P. Bartel: Metazoan MicroRNAs. Cel/2018, 173(1):20-51.

40. L. Wang, X. Chen, Y. Zheng,et al: MiR-23a inhibits myogenic differentiation through down regulation of fast myosin heavy chain isoforms. Experimental Cell Research2012, 318(18):2324-2334.

Page 10/18 
41. W. Shogo, K. Yoshio, O. Mitsuharu, et al: Translational suppression of atrophic regulators by microRNA-23a integrates resistance to skeletal muscle atrophy. The Journal of biological chemistry2011, 286(44):38456-38465.

42. C. Mengjie, W. Xuefeng, S. Mingming, et al: Circular RNA circMYBPC1 promotes skeletal muscle differentiation by targeting MyHC. Molecular Therapy - Nucleic Acids2021, 24:352-368.

43. W.C. FaiandT.R. L: MicroRNA-26a targets the histone methyltransferase Enhancer of Zeste homolog 2 during myogenesis. The Journal of biological chemistry2008, 283(15):9836-9843.

\section{Figures}

DAPI
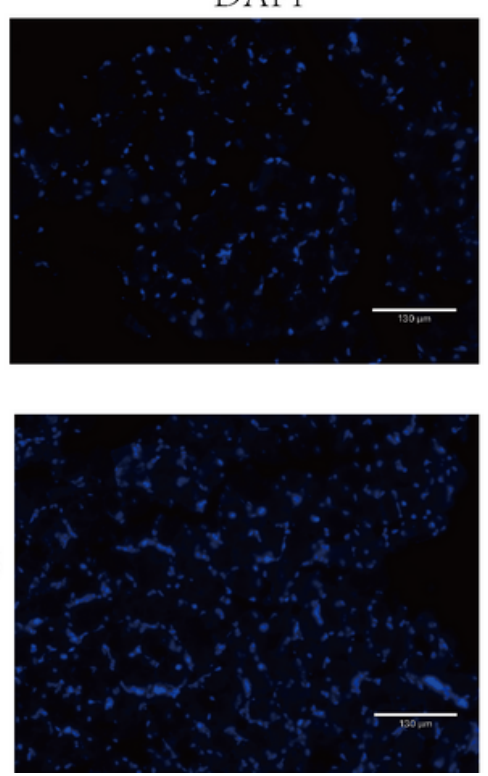

MYH7
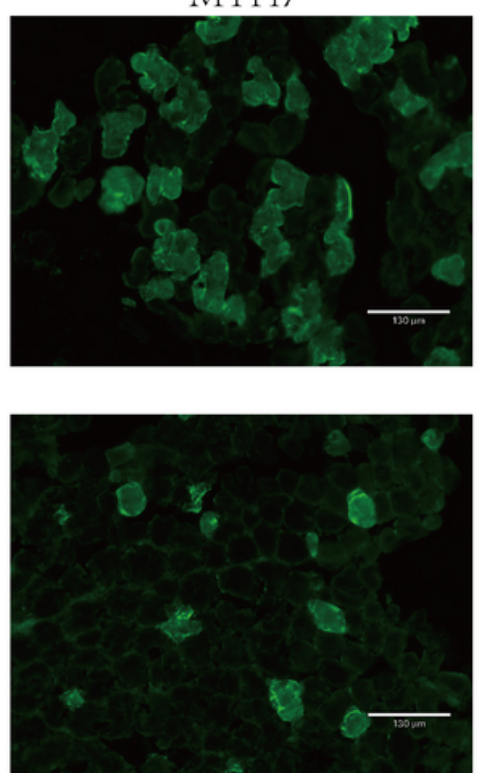

Merge
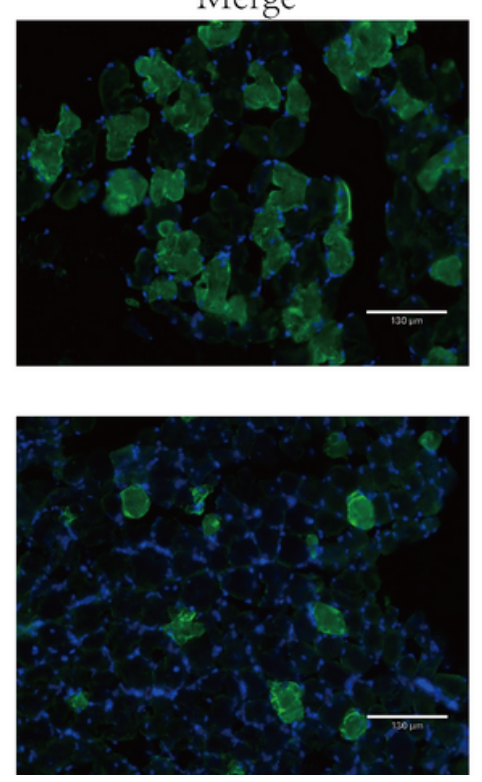

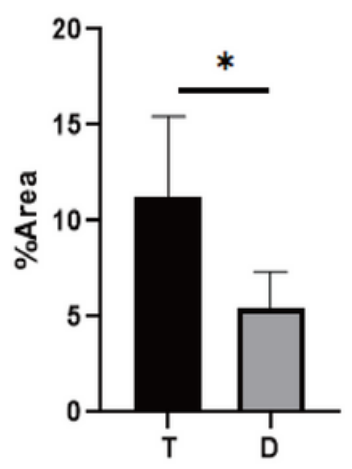

Figure 1

The difference between the slow-twitch fiber ratio of the longissimus dorsi muscle of Tan sheep and Dorper sheep was observed and counted by frozen section $\mathrm{MyH} 7$ immunofluorescence staining. 
a.

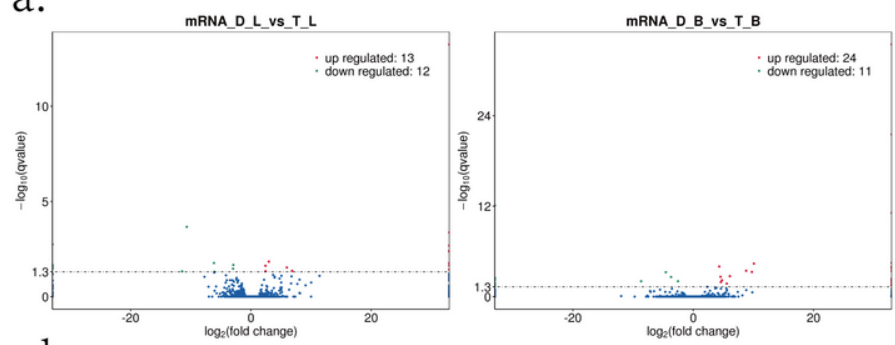

b.

Enriched Go Terms
(D_L_vs_T_L)

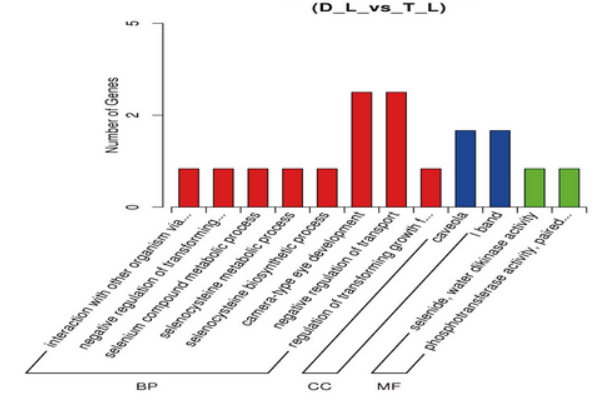

C.

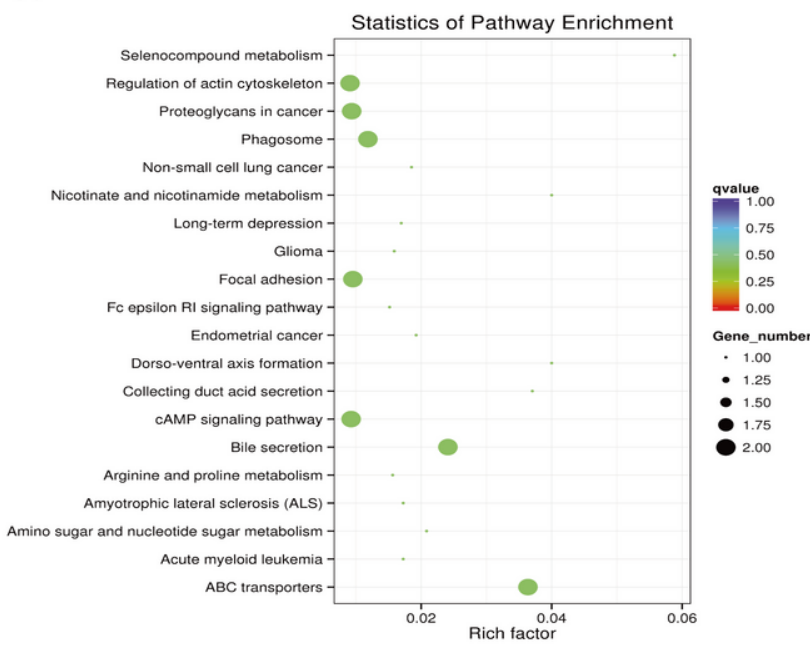

Figure 2

Differentially expressed mRNAs in LD and BF muscle in three groups. a. Volcano Plot pictures showing log ${ }_{2} \mathrm{FC}$ values and $q$ value of IncRNAs in D_L/T_L and D_B/T_B. The red dots and green dots respectively represent up-regulated and down-regulated mRNAs during muscle fibers formation. b. GO enrichment of targets of DE IncRNAs in D_L/T_L. C. KEGG enrichment of targets of DE IncRNAs in D_L/T_L. Only the top 20 enriched pathways are presented here. 

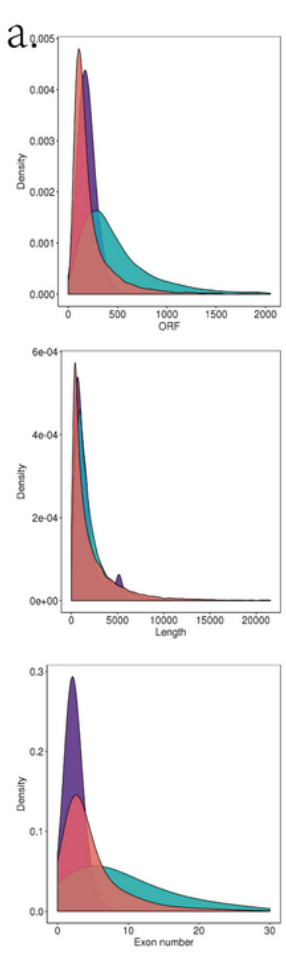

d.
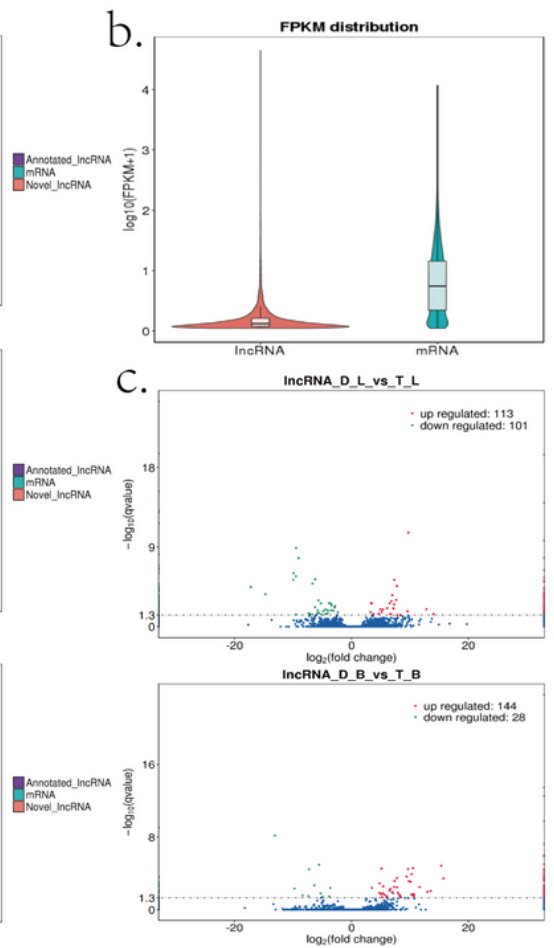

e.

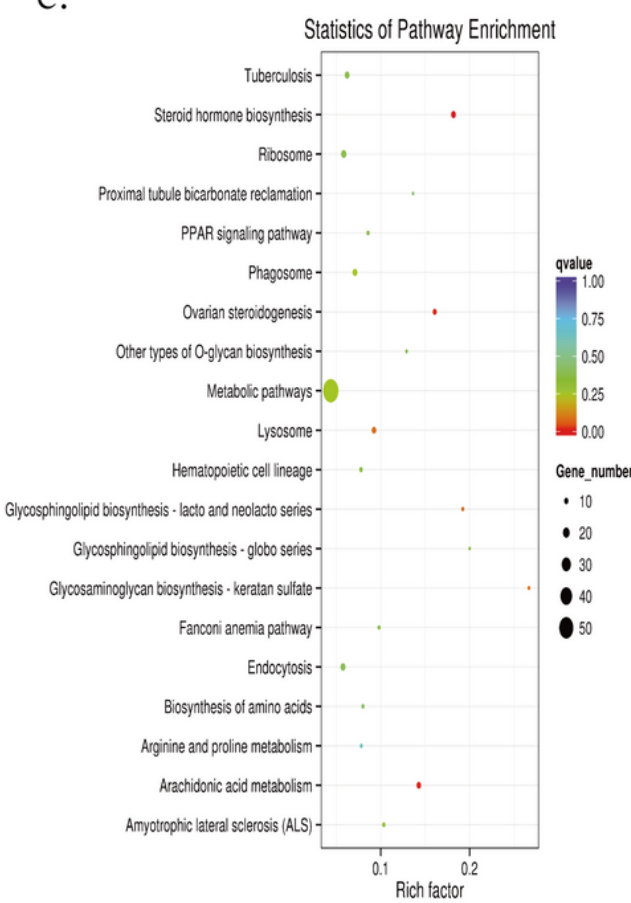

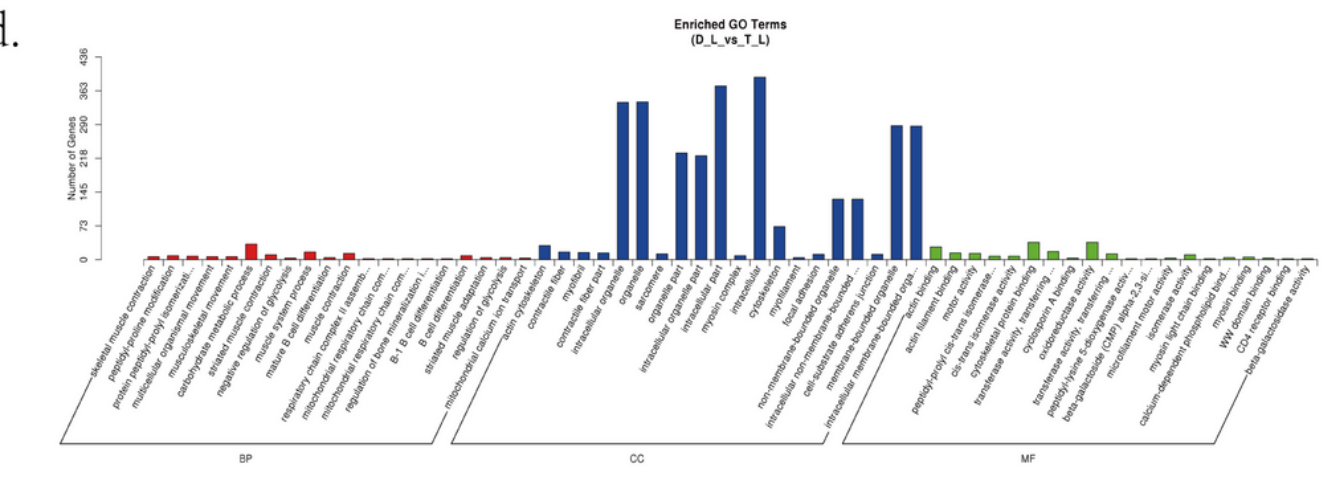

Figure 3

Differentially expressed IncRNAs in LD and BF muscle in three groups. a. Comparison of IncRNA with mRNA with respect to the transcript length, exon number and ORF length. b. Comparison of IncRNA with mRNA with respect to the FPKM value. c. Volcano Plot pictures showing $\log _{2} \mathrm{FC}$ values and $q$ value of IncRNAs in D_L/T_L and D_B/T_B. d. GO enrichment of targets of DE IncRNAs in D_L/T_L. e. KEGG enrichment of targets of $D E$ IncRNAs in D_L/T_L. Only the top 20 enriched pathways are presented here. 


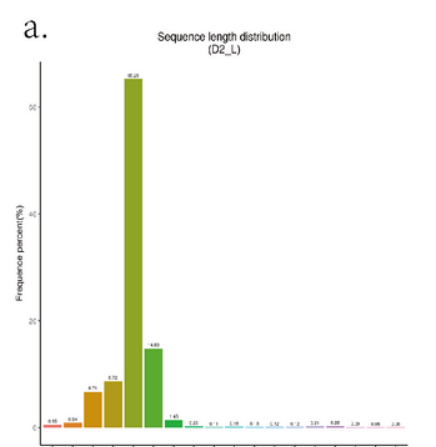

b.

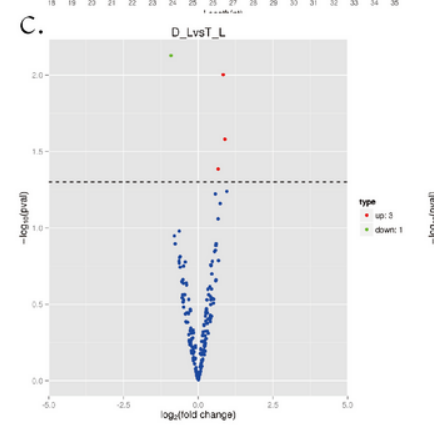

d.

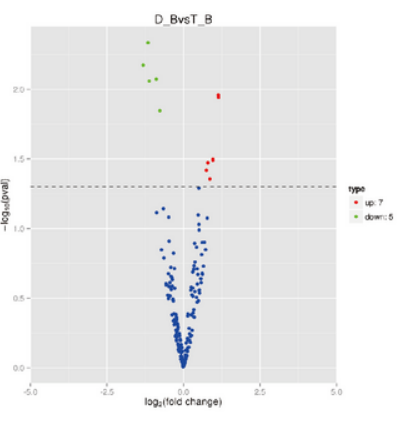

Enriched GO Terms
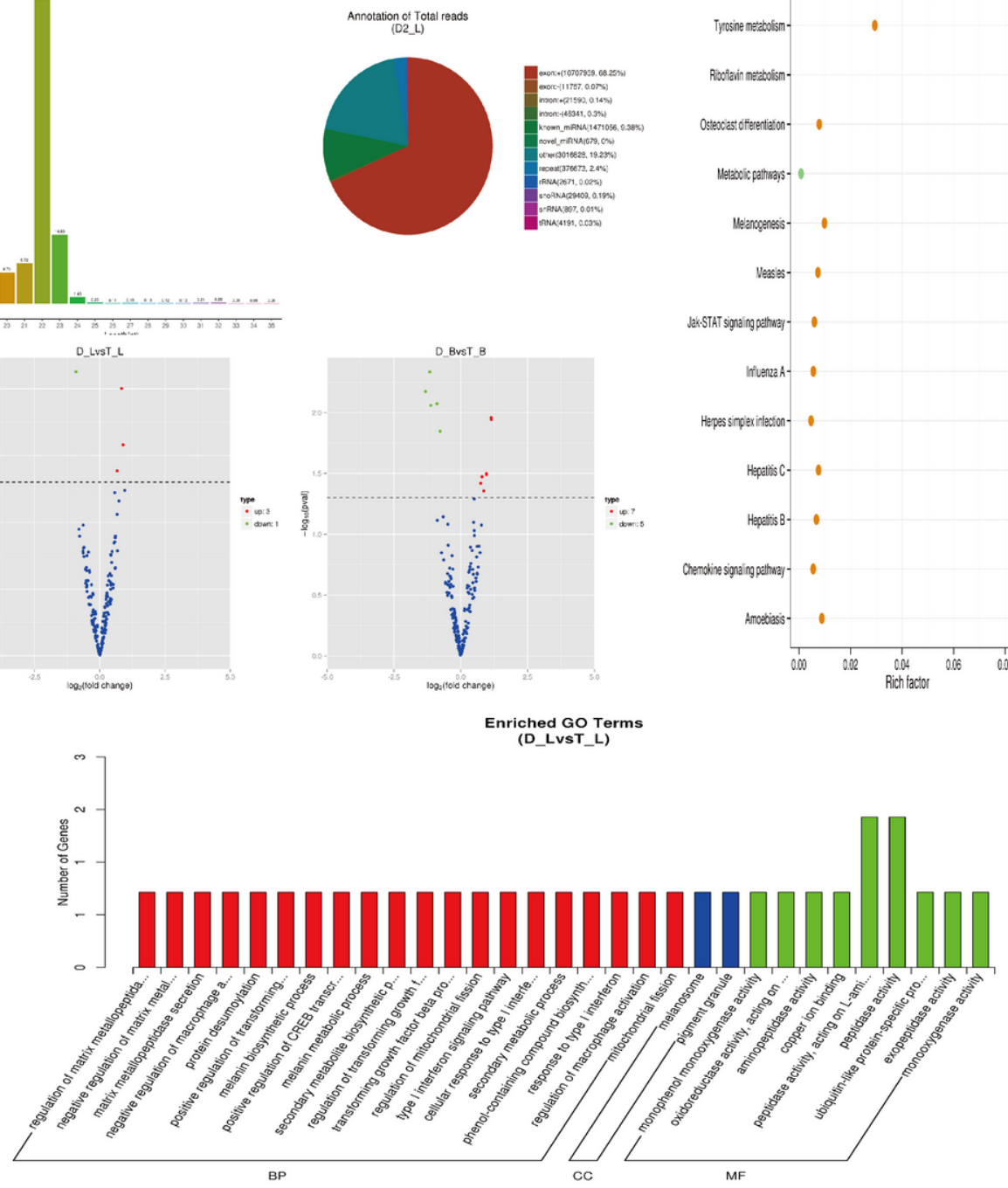

e.

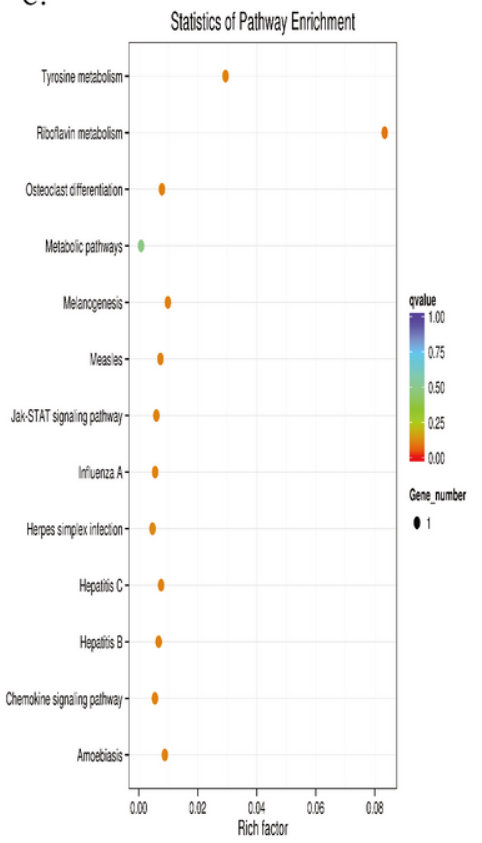

\section{Figure 4}

Differentially expressed miRNAs in LD and BF muscle in three groups. a. Length distribution of all identified small RNAs. Percentage of different types of small RNAs. b. Percentage of different types of small RNAs. c. Volcano Plot pictures showing $\log _{2} F C$ values and $q$ value of miRNAs in D_L/T_L and D_B/T_B. d. GO enrichment of targets DE miRNAs in D_L/T_L. e. KEGG enrichment of targets DE miRNAs in D_L/T_L. 


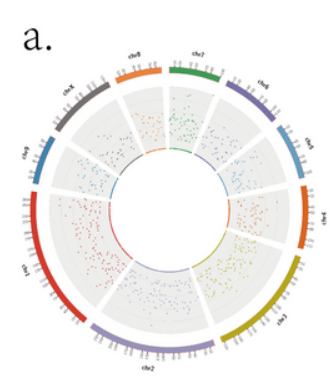

b.

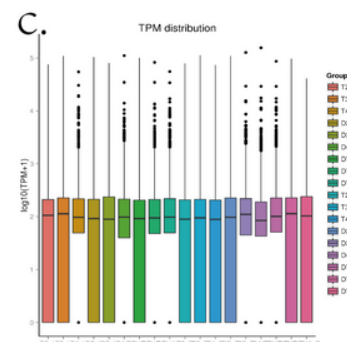

d.
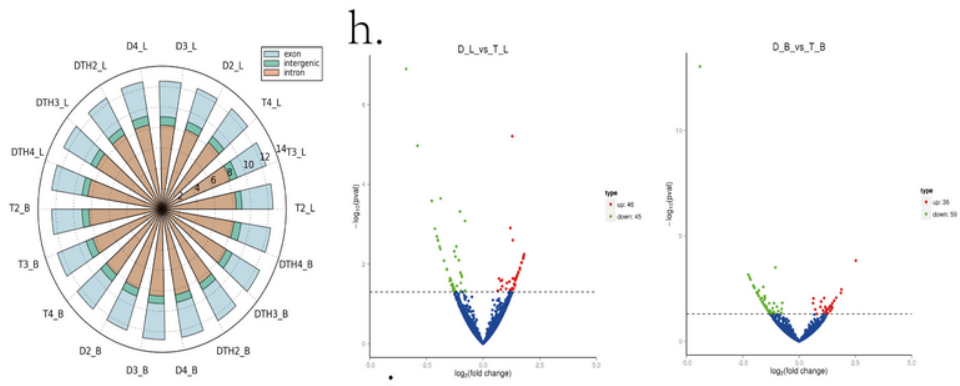

i.

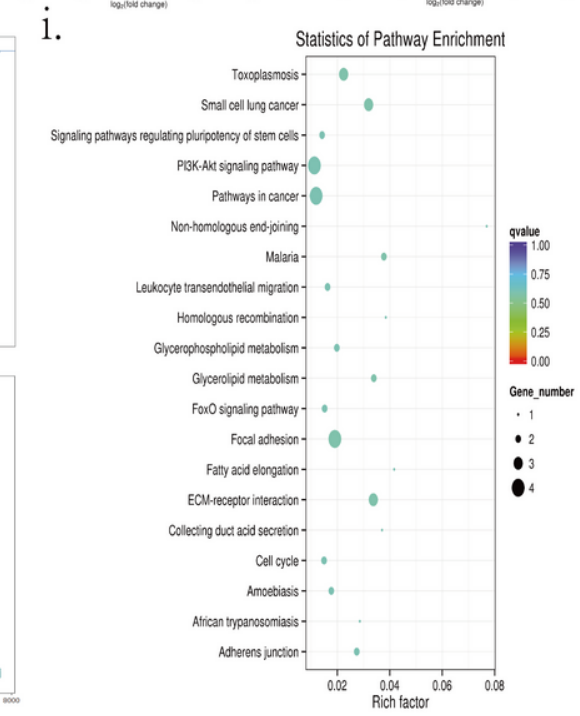

g.

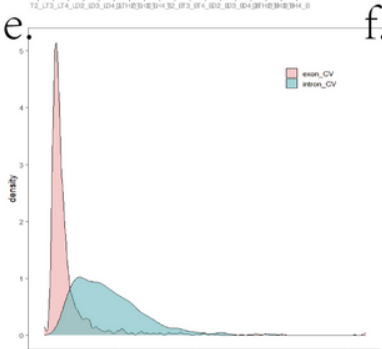

f.
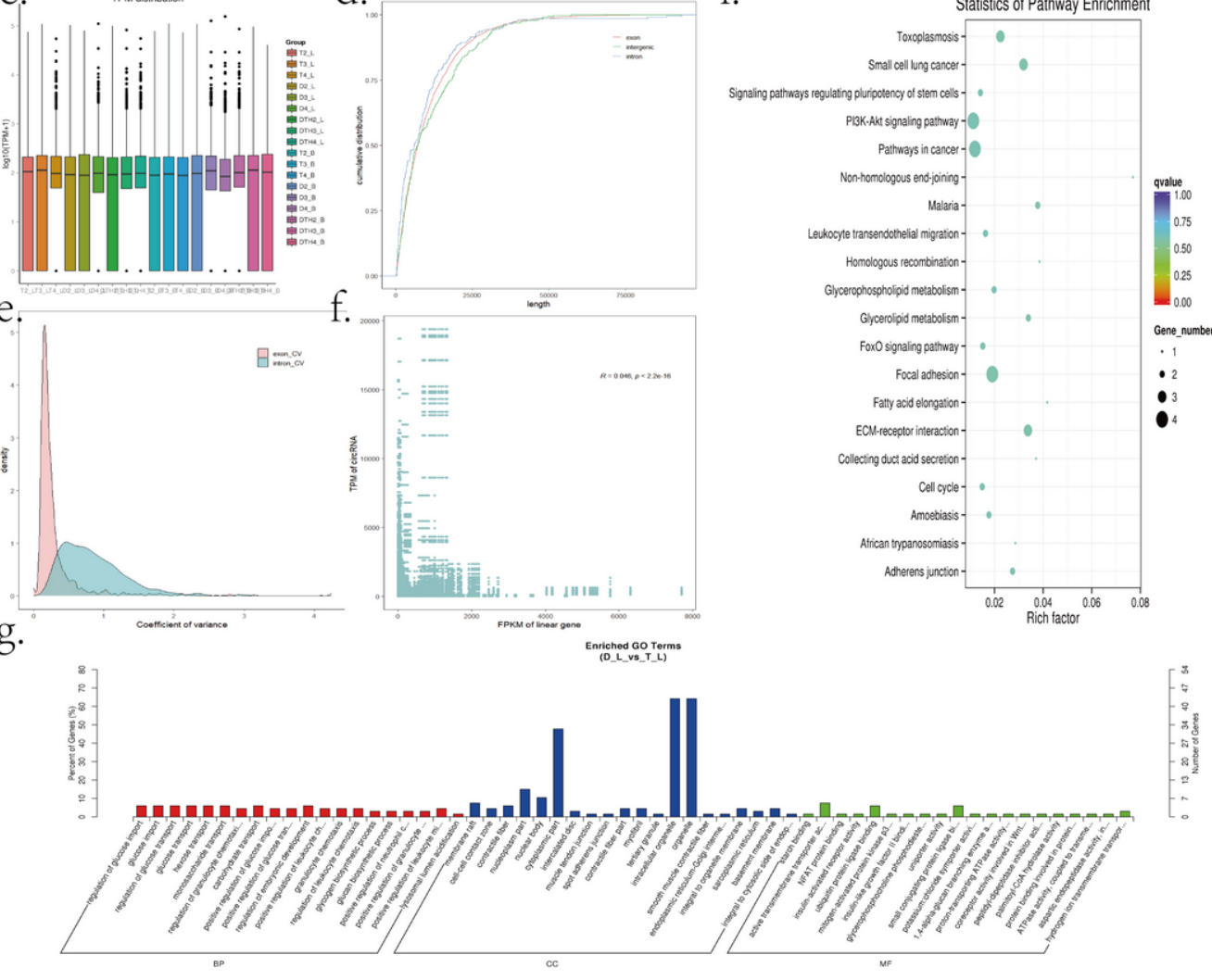

Figure 5

Differentially expressed ciRNAs in LD and BF muscle in three groups. a. The proportion of different categories of circRNAs in sheep. b. The distribution of novel circRNAs in different chromosomes. c. Box plot of circRNA expression level of each sample, and normalized expression level with TPM. d. Cumulative distribution of three different types of circRNAs length. e. Expression correlation between circRNAs and their respective parental genes. $f$. Expression variance of circRNAs and their parental genes. h. Volcano Plot pictures showing $\log _{2} \mathrm{FC}$ values and $q$ value of miRNAs in D_L/T_L and D_B/T_B. g. GO enrichment of targets DE miRNAs in D_L/T_L. i. KEGG enrichment of targets DE miRNAs in D_L/T_L. 


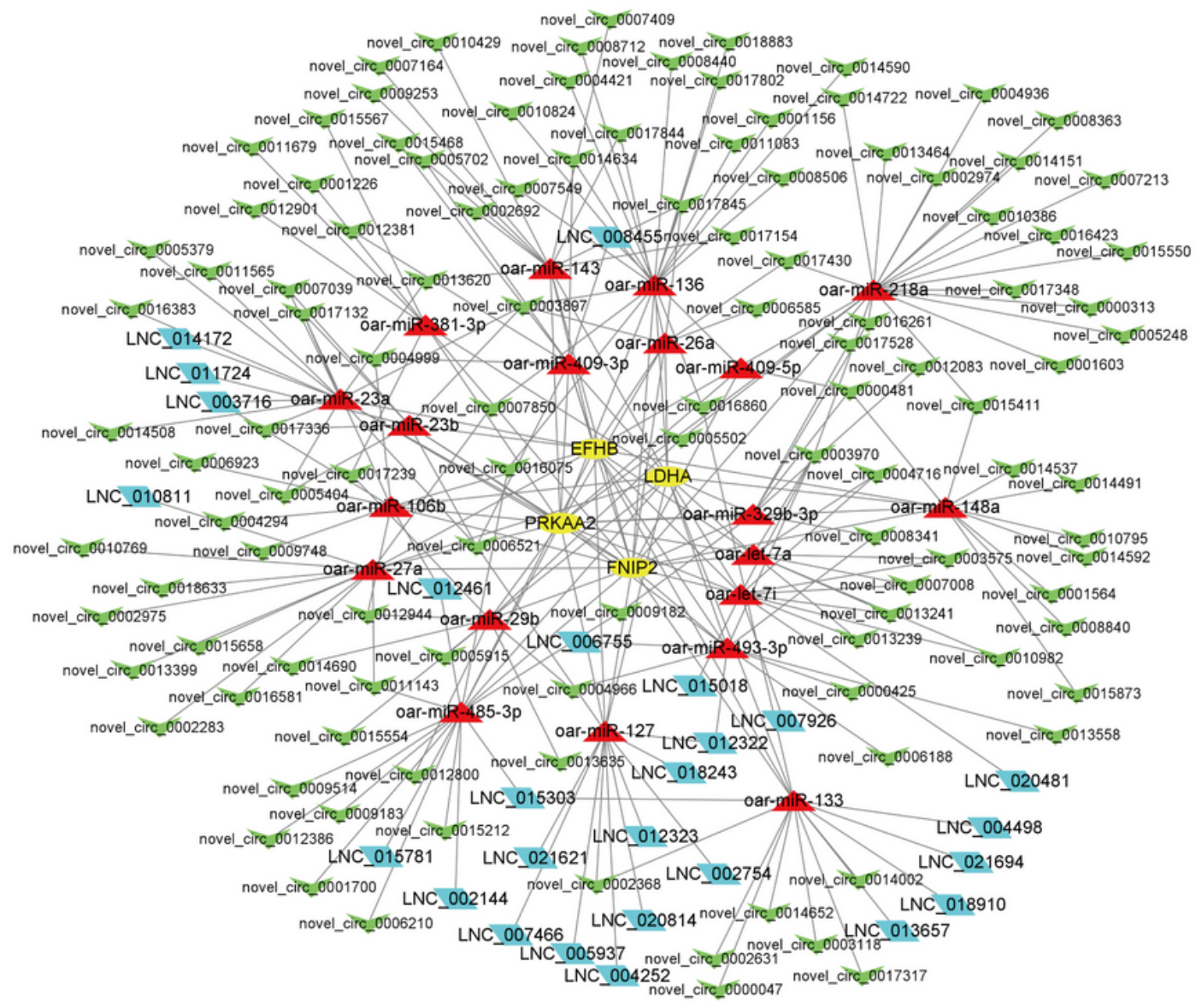

Figure 6

LncRNA/circRNA-miRNA-mRNA interaction network. ovals, triangle, parallelogram and $V$ type represent mRNAs, miRNAs, IncRNAs and circRNAs, respectively. 

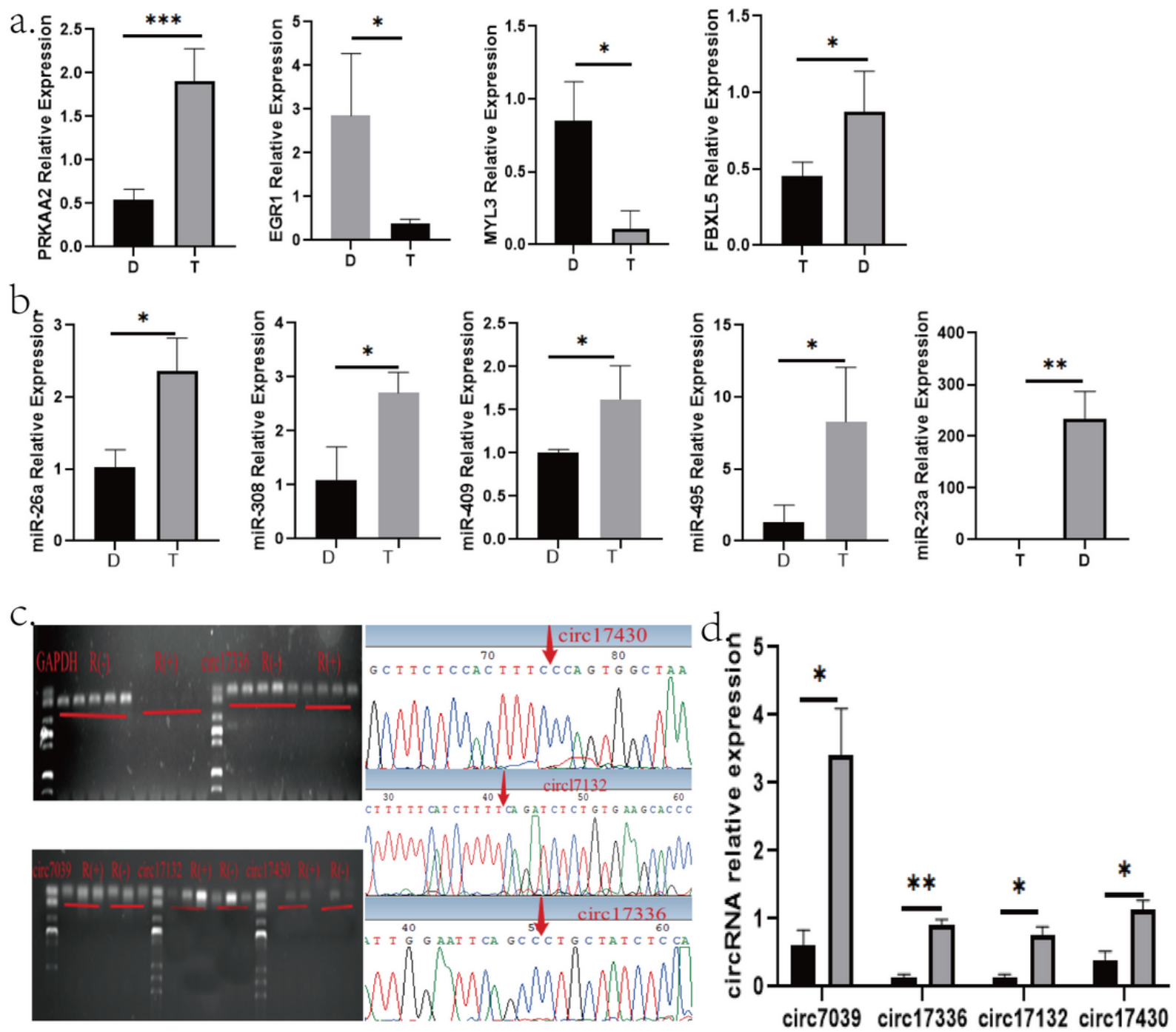

\section{Figure 7}

The validation of the mRNA, miRNA and circRNA RNA-seq results. a. the PRKAA2, EGR1, MYL3 and FBXL5 mRNA expression level. b. the miR26a, miR-308-3p, miR-409-3p, miR-495-3p and miR-23a expression level. c.circ_0017336, circ_0017132, circ_0017430, and circ_0007039 were amplified with RNase R and the Sanger sequencing. "R+" indicates RNA treated with RNase R; "R-"indicates untreated RNA. The red arrows indicate head-to-tail back-splicing sites of circRNAs. d. the circ_0017336, circ_0017132, circ_0017430, and circ_0007039 circRNA expression level.

\section{Supplementary Files}

This is a list of supplementary files associated with this preprint. Click to download.

- TableS1.OverviewofthedataforRNAandsmallRNAsequencing.xlsx

- TableS12.PrimersequencesforqPCR..xlsx

- TableS8.DifferentiallyexpressedmiRNAsinLDandBFmuscleinthreegroups..xlsx

- TableS3.DifferentiallyexpressedmRNAsinLDandBFmuscleinthreegroups..xlsx

- TableS5.DifferentiallyexpressedIncRNAsinLDandBFmuscleinthreegroups..xlsx

- TableS4.GOenrichmentandKEGGpathwayanalysisforDEmRNAs..xIsx

- TableS10.GOenrichmentandKEGGpathwayanalysisforDEcircRNAsparentalgenes..xlsx

- TableS22.IdentifiedtissuespecificexpressedmRNAsinourdata..xIsx 
- TableS6.GOenrichmentandKEGGpathwayanalysisforDEcisIncRNAstargetgenes..xlsx

- TableS11.IRESanalysisresultsofcircRNAsequence..xIsx

- TableS9.GOenrichmentandKEGGpathwayanalysisforDEmiRNAstargetgenes..xIsx

- TableS21.IdentifiedcircRNAsinourdata..xlsx

- Fig.S3VenndiagramshowingthenumberofcodifferentlyexpressedmRNAalncRNAbmiRNAccircRANdinbothLDandBFtissuesinthreegroups..tif

- TableS7.GOenrichmentandKEGGpathwayanalysisforDEtransIncRNAstargetgenes..xlsx

- Fig.S1VenndiagramshowingthenumberoflncRNAsidentifiedbyCNCICPCandPfamScanmethods..tif

- Fig.S5gelsdigitalimage.Theexpressionofcirc7039circ17132andcirc17430withoutorwithRnaseRtreatment..pdf

- Fig.S2LncRNAcircRNAmiRNAmRNAinteractionnetworkcenteredonLDHAaFNIP2bPRKAA2cEFHBd..tif

- Fig.S4gelsdigitalimage.TheexpressionofGAPDHandcirc17336withoutorwithRnaseRtreatment..pdf 